\title{
Backhoe-assisted monolith method for plant root phenotyping under upland conditions
}

\author{
Shota Teramoto(1), Yuka Kitomi ${ }^{1)}$, Ryo Nishijima ${ }^{2)}$, Satoko Takayasu' ${ }^{1)}$, Natsuko Maruyama ${ }^{1)}$ and Yusaku Uga*1) \\ 1) Institute of Crop Science, National Agriculture and Food Research Organization, Tsukuba, Ibaraki 305-8518, Japan \\ 2) Institute of Agrobiological Sciences, National Agriculture and Food Research Organization, Tsukuba, Ibaraki 305-8602, Japan
}

\begin{abstract}
Root system architecture (RSA) is one of the most important traits determining water and nutrient availability for plants. Modification of RSA is known to be a useful approach for improving root performance of crops. However, for conducting root phenotyping, there are few alternatives for the rapid collection of root samples from a constant soil volume. In this report, we propose a rapid root-sampling method, which uses a steel cylinder known as round monolith and backhoes to reduce the physical effort. The monolith was set on the ground surrounding individual rice plants and vertically driven back by a backhoe. Soil samples with $20 \mathrm{~cm}$ width and $25 \mathrm{~cm}$ depth were excavated by the monolith, from which root samples were then isolated. This backhoeassisted monolith method requires at most five minutes to collect root samples from one plant. Using this method, we quantified the root traits of three rice lines, reported to form different types of root system such as shallow-, intermediate-, and deep-roots, using a root image analysis software. The data obtained through this method, which showed the same trend as previously reported, clearly demonstrated that this method is useful for quantitative evaluation of roots in the soil.
\end{abstract}

Key Words: monolith, rice, root morphology, root sampling, image analysis.

\section{Introduction}

Root system architecture (RSA) is one of the most important plant traits, as roots not only anchor plant body to soil but also determine its nutrient availability (Smith and De Smet 2012). Modifications of RSA would minimize the negative impact of abiotic stress on crop yield; for that reason, many genes and quantitative trait loci (QTLs) affecting RSA have been identified for crop improvement (de Dorlodot et al. 2007).

RSA is defined as the morphological and functional differences in root deployment in the soil (Lynch 1995). In cereals including rice (Oryza sativa L.), one radicle (primary root) firstly emerges after germination, followed by many crown roots (adventitious roots) that emerge from basal stem nodes (Hochholdinger et al. 2004, Itoh et al. 2005, Yoshida and Hasegawa 1982). Radicle and crown roots develop lateral roots, which play a key role in water and nutrient uptake (Atkinson et al. 2014). In other words, crown and lateral roots contribute to the rhizosphere area and the roots density in the soil, respectively. For example, the modifica-

Communicated by Manabu Ishitani

Received February 4, 2019. Accepted May 4, 2019.

First Published Online in J-STAGE on July 10, 2019.

*Corresponding author (e-mail: yuga@affrc.go.jp) tion of the RSA with the DEEPER ROOTING 1 (DRO1) gene increased rice yield under drought conditions by expanding the rhizosphere deeper (Uga et al. 2013). Another example of RSA relevance arises from simulation analyses carried out in maize, which revealed that phosphorous uptake is optimized by dense and short lateral roots, whereas nitrate uptake is optimized by sparse and long lateral roots (Postma et al. 2014).

Roots being underground organs, root phenotyping under field conditions is very laborious and time-consuming (Böhm 2012). Thus, root phenotyping systems in laboratories or artificially-controlled conditions have been widely used for the high-throughput of root characterization. For example, hydroponic culture is one of the cultivation systems that allow easy root sampling and phenotyping. Rhizobox, a kind of transparent culture box for root phenotyping, is also popular because root traits are easy to observe and measure. Both systems could deal with many samples at the same time, which is necessary for QTL mapping and genome-wide association studies (e.g. Courtois et al. 2013, Kitomi et al. 2018, Slovak et al. 2014, Song et al. 2016, Uga et al. 2018, Zhao et al. 2018). Since these methods involve artificial growth conditions, procedures allowing root phenotyping under real field conditions remain as a research need.

'Shovelomics' is a field root phenotyping approach based 
on root sampling by digging up the roots with shovels, followed by a visual scoring that allows root phenotyping (Trachsel et al. 2011). Due to its simplicity and highthroughput, shovelomics is one of the most popular methods for root phenotyping of field-grown plants. However, shovelomics is not suitable to the quantification of root traits because it uses shovels for digging up the roots, which may involve root loses or hurting, and employs a visual scoring method, which is semi-quantitative.

For collecting and measuring root samples, soil-core sampling and monolith methods are mainly used (Böhm 2012). Soil coring drives small-diameter cylinders into the ground vertically to collect soil samples, from which root samples are isolated and quantified (Wasson et al. 2014). The procedure is simple and easy, but the sampling area is limited. The monolith method drives relatively large boxes or cylinders with bottom side open into the ground vertically to collect soil block containing root samples (Wu and Guo 2014). The box and cylinder are conventionally known as 'monolith' and 'round monolith', respectively. Both soil coring and monolith methods requires hammers or custombuilt machines to drive the core samplers or monoliths into the ground, indicating that soil coring and monolith methods are more complicated and laborious more than shovelomics may.

Modern cultivation uses a lot of fertilizers to increase crop yields (Food and Agriculture Organization of the United Nations 2015), and the fertilizers are mainly distributed in the plow layer. Most of roots are also concentrated in the plow layer although the distribution of roots depends on subsoil tillage (Cai et al. 2014). Therefore, we considered that a rapid sampling method for roots growing in the plow layer, suitable for quantitative root phenotyping, would be helpful not only for the characterization of RSA of crops but also the breeding of new cultivars. In this regard, the monolith method is one of the most suitable ones for quantifying root traits in the plow layers. However, the monolith method is laborious and time-consuming compared with shovelomics.

In this study, we present a rapid sampling method for plant root phenotyping, based on the use of a round monolith and backhoes, to reduce physical effort. Using this method, we collected rice roots from a constant volume of the plow layer, taking the operation five minutes per sample. Furthermore, we quantified root traits of three cultivars or lines using a root image-analysis software and corroborated the difference in root characters related to RSA between indica and tropical japonica cultivars.

\section{Materials and Methods}

\section{Plant materials}

Three rice (Oryza sativa) cultivars or lines, 'IR64' (IRGC \#66970), 'Kinandang Patong' (KP, IRGC \#23364), and 'Dro1-NIL' (Uga et al. 2013), were used in this study. IR64 is a lowland indica cultivar and KP is an upland tropical japonica cultivar. It was previously reported that KP has thicker, longer, and steeper roots than IR64 (Kondo et al. 2000, Uga et al. 2018), which is a typical character of upland rice. Dro1-NIL is a near-isogenic line of IR64 genetic background with the KP allele of DRO1 gene, which has been identified as a gene controlling root growth angle by QTL cloning in a mapping population derived from a cross between IR64 and KP (Uga et al. 2013). IR64, KP, and Dro1-NIL show monomorphic shallow-, monomorphic deep-, and dimorphic-rooting patterns, respectively.

\section{Field and experimental design}

We cultivated rice plants during the summer season of 2018 at an upland field of the Institute of Crop Science (National Agriculture and Food Research Organization, Ibaraki, Japan; $36^{\circ} 02^{\prime} 89^{\prime \prime} \mathrm{N}$ and $\left.140^{\circ} 09^{\prime} 97^{\prime \prime} \mathrm{E}\right)$. The soil at the experimental site is a volcanic ash soil of the Kanto loam type (Humic Andosol). The topsoil $(0-30 \mathrm{~cm})$ is a dark humic silty loam. The subsoil (below $30 \mathrm{~cm}$ ) is a redbrown silty clay loam. There was a hardpan at a depth of about 20 to $25 \mathrm{~cm}$. The field was fertilized with $5.2 \mathrm{~g} \mathrm{~N} \mathrm{~m}^{-2}$, $15.4 \mathrm{~g} \mathrm{P}_{2} \mathrm{O}_{5} \mathrm{~m}^{-2}$, and $5.6 \mathrm{~g} \mathrm{~K}_{2} \mathrm{O} \mathrm{m}^{-2}$ before rice planting. We employed a block design for evaluating rice traits. Three blocks were arranged as three replications. Each block included three plots for IR64, KP, and Dro1-NIL, and contained 15 hills spaced by $0.4 \mathrm{~m} \times 0.4 \mathrm{~m}$. Three hills were used for monolith sampling, while three other hills were used for the basket assay. Three seeds were sown in each hill on June 5. On July 3 and July 4, we adjusted seedling density to one seedling per hill by removing seedlings with growth defects and transplanting those with intermediate growth. Simultaneously, three plastic mesh baskets $(15 \mathrm{~cm}$ diameter and $6 \mathrm{~cm}$ height) were filled with soil and buried at certain hills in each plot for basket assay. Water was supplied with a sprinkler before starting leaf rolling of rice seedlings.

\section{Monolith design}

The monolith was designed as a steel cylinder $(20 \mathrm{~cm}$ diameter and $30 \mathrm{~cm}$ depth), tough enough to be driven from the ground by a backhoe. Although the plow layer thickness depends on several factors including crop rotations, climate, and schedule of planting, the depth of an upland rice plow layer is $25 \mathrm{~cm}$ at most (Gupta and O'Toole 1986). Thus, the monolith we developed is suitable for the evaluation of rice root properties in the plow layers. An external view of the round monolith and an unfolded scheme are shown in Fig. 1, respectively. The thickness of the monolith wall was $8.2 \mathrm{~mm}$, and a steel disk was welded to the top side to improve its mechanical properties. The bottom side was sharpened to sever roots when monolith was driven into the ground. Four equidistant holes were drilled at $3 \mathrm{~cm}$ from the top side for craning the monolith from the soil.

\section{Backhoe-assisted monolith sampling}

The tiller number and height of the plants selected for monolith sampling were measured on July 25. Above- 

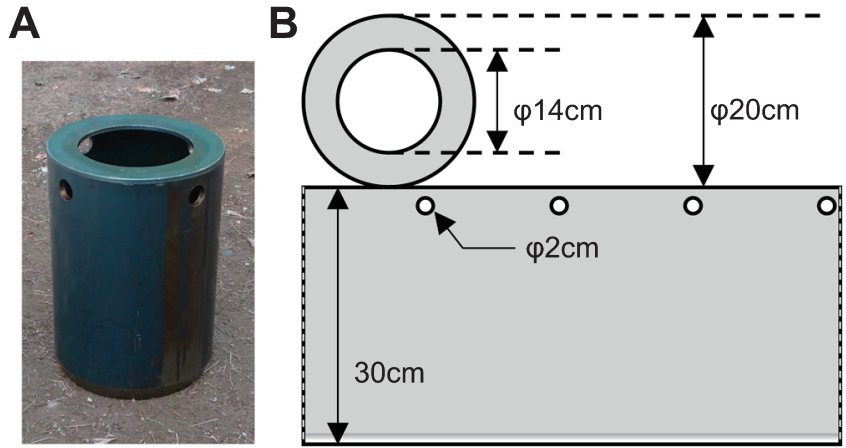

Fig. 1. Round monolith used in this study. (A) Image of the monolith. (B) Unfolded scheme of the monolith. All diameters are shown as inside diameters. The thickness of the wall was $8.2 \mathrm{~mm}$. A steel disk was welded to the top side of the monolith to improve its mechanical properties.

ground tissues from the three blocks were sampled on July 26, July 30, and August 1 , in coincidence with the days of monolith sampling. The above-ground tissues were dried at $80^{\circ} \mathrm{C}$ for one week to measure their dry weight. Around late July and early August are the tillering stage for IR64, KP, and Dro1-NIL, the period during which rice plants increasing their tiller number and biomass. Since the tillering stage is one of the most important periods affecting rice yield (Fageria 2007), we collected shoot and root samples during these periods.

Rice roots were sampled with the monolith and backhoes. The monolith was set up on the ground with the plant base as the horizontal center and driven into the soil vertically by pushing the upper side of the monolith with the blade of the B27 backhoe (YANMAR, Japan) to a depth of $25 \mathrm{~cm}$ (Fig. 2A, 2B). The monolith containing soil and root samples was excavated by craning from the ground by the backhoe (Fig. 2C, 2D). Soil and root samples were detached from the round monolith with a RA400 loader (KUBOTA, Japan) by pushing the upper side of the sample (Fig. 2E, 2F). A more detailed procedure is shown in Supplemental Fig. 1.

Root samples were washed with water to remove soil and dust and stored in $70 \%$ ethanol at $10^{\circ} \mathrm{C}$ until use. The volume of each hole after excavating was $7,850 \mathrm{~cm}^{3}(20 \mathrm{~cm}$ diameter and $25 \mathrm{~cm}$ depth).

\section{Basket assay}

The ratio of deep rooting (RDR) was measured on July 24 with the basket assay, according to a previous study (Uga et al. 2009). All baskets were dug up and subjected to manual counting of the roots that penetrated the holes. The RDR was calculated by dividing the total number of roots penetrating the lower part of the mesh (53 to $90^{\circ}$ to a horizontal axis) by the total number of roots penetrating the whole mesh.

\section{Root image analysis}

Total root length, total root surface area, total root vol-
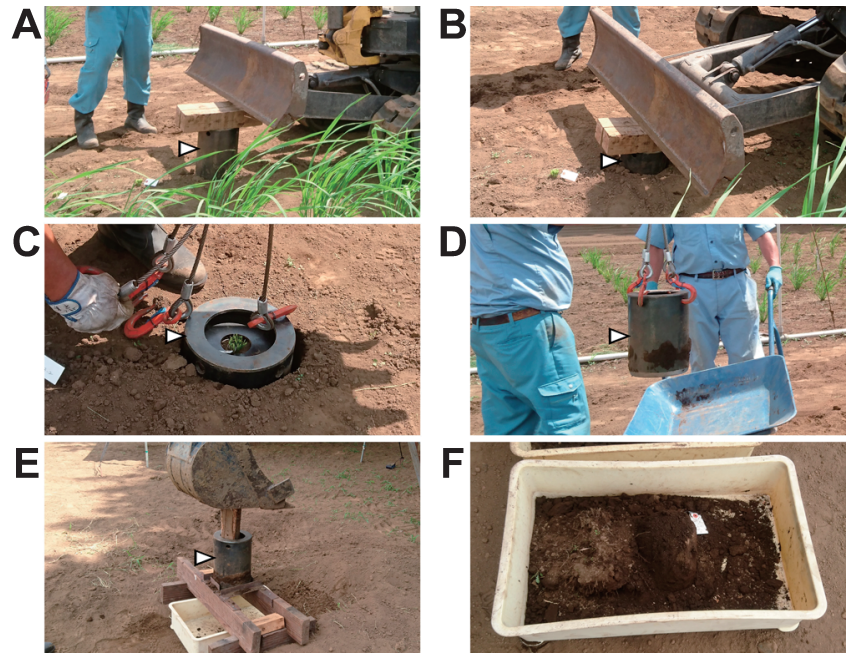

Fig. 2. Rice root sampling with backhoes. (A) The monolith was set up on the stump. Large tree blocks were placed between the monolith and the backhoe as cushion. A small steel disk was set on the soil in the monolith, which was used for effective isolation of the soils. The disk can be confirmed in panel C. (B) The monolith was driven into the soil vertically with the blade of the backhoe. (C) Hook wires were connected with the monolith and backhoe. Four hooks were inserted into the holes of the monolith. (D) The monolith containing soil and root samples was excavated by craning. (E) The sample was detached from the round monolith by pushing the upper side of the sample with the loader and a large tree block. (F) Soils with root samples were collected in the box. Arrowheads indicate the monolith.

ume, and root diameter were measured with WinRHIZO ${ }^{\mathrm{TM}}$ Pro 2017a software (Regent Instruments, Canada). Crown roots were separated by cutting and rinsed with reverseosmosis water, and all roots were spread out on the tray for scanning. The scanning resolution was $400 \mathrm{dpi}$. According to their diameters, roots were divided into two classes: less than $0.2 \mathrm{~mm}$ and over $0.2 \mathrm{~mm}$. Since it is known that the diameter of lateral roots in rice is less than $0.2 \mathrm{~mm}$ (Henry et al. 2012), the former class includes lateral roots and the latter class includes radicle and crown roots. The average root diameter of each class was calculated as:

$$
D=2 \sqrt{\frac{V}{\pi L}}
$$

where D is root diameter $(\mathrm{cm}), \mathrm{V}$ is total root volume $\left(\mathrm{cm}^{3}\right)$, and $\mathrm{L}$ is total root length $(\mathrm{cm})$. All data were processed with the R software (https://www.r-project.org/). After scanning, roots were dried at $80^{\circ} \mathrm{C}$ for three days to measure their dry weight.

\section{Statistical analysis}

Average within each block was calculated, these data were used for statistical analysis. Dunnett's test against IR64 was performed with 'multcomp' package (version 1.4.8) in R software. This study defined that comparisons with $P$ values under 0.05 entail significant difference. 


\section{Results}

Root phenotyping using the backhoe-assisted monolith method

Roots of IR64, Dro1-NIL, and KP were collected by the backhoe-assisted monolith method. The shapes of the whole roots including shoot bases are shown in Fig. 3A-3C. Whole roots in the plow layer were successfully collected. All crown roots were separated from each other by removing the shoot base and subjected to scanning (Fig. 3D-3F). Compared with IR64 and Dro1-NIL, KP had a larger diameter of crown roots and a lower number of crown and lateral roots, i.e., shorter total root length. On the other hand, there were no apparent differences between IR64 and Dro1-NIL.

To compare root traits quantitatively, scan images were analyzed with the WinRHIZO ${ }^{\mathrm{TM}}$ software. Total lengths of both crown and lateral roots in KP were significantly lower than those in IR64 (Fig. 4A, 4B), indicating that root density of KP in the plow layer was sparse. Because crown roots in rice sprang out from nodes (Atkinson et al. 2014, Yoshida and Hasegawa 1982), one of the reasons for lower root density of KP was lower tiller number (Supplemental Fig. 2A). Total lengths of both crown and lateral roots in Dro1-NIL were also lower than those of IR64, though not significantly
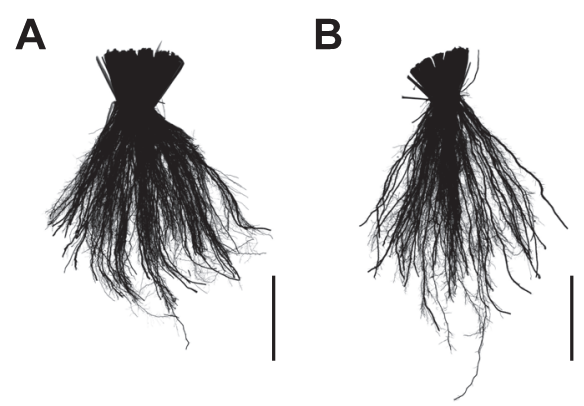

D

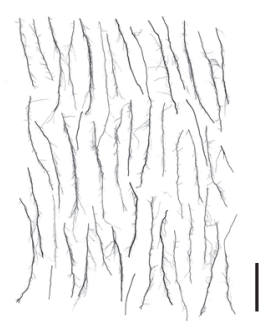

E

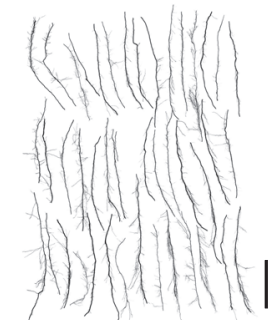

$\mathbf{F}$
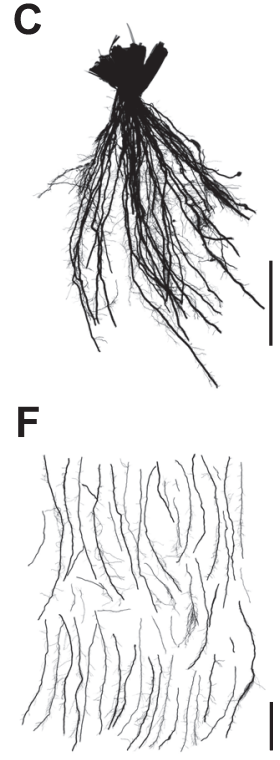

Fig. 3. Representative scan images of whole root samples; (A) IR64, (B) Dro1-NIL, and (C) KP. Images of separated roots; (D) IR64, (E) Dro1-NIL, and (F) KP. Bars indicate $5 \mathrm{~cm}$.
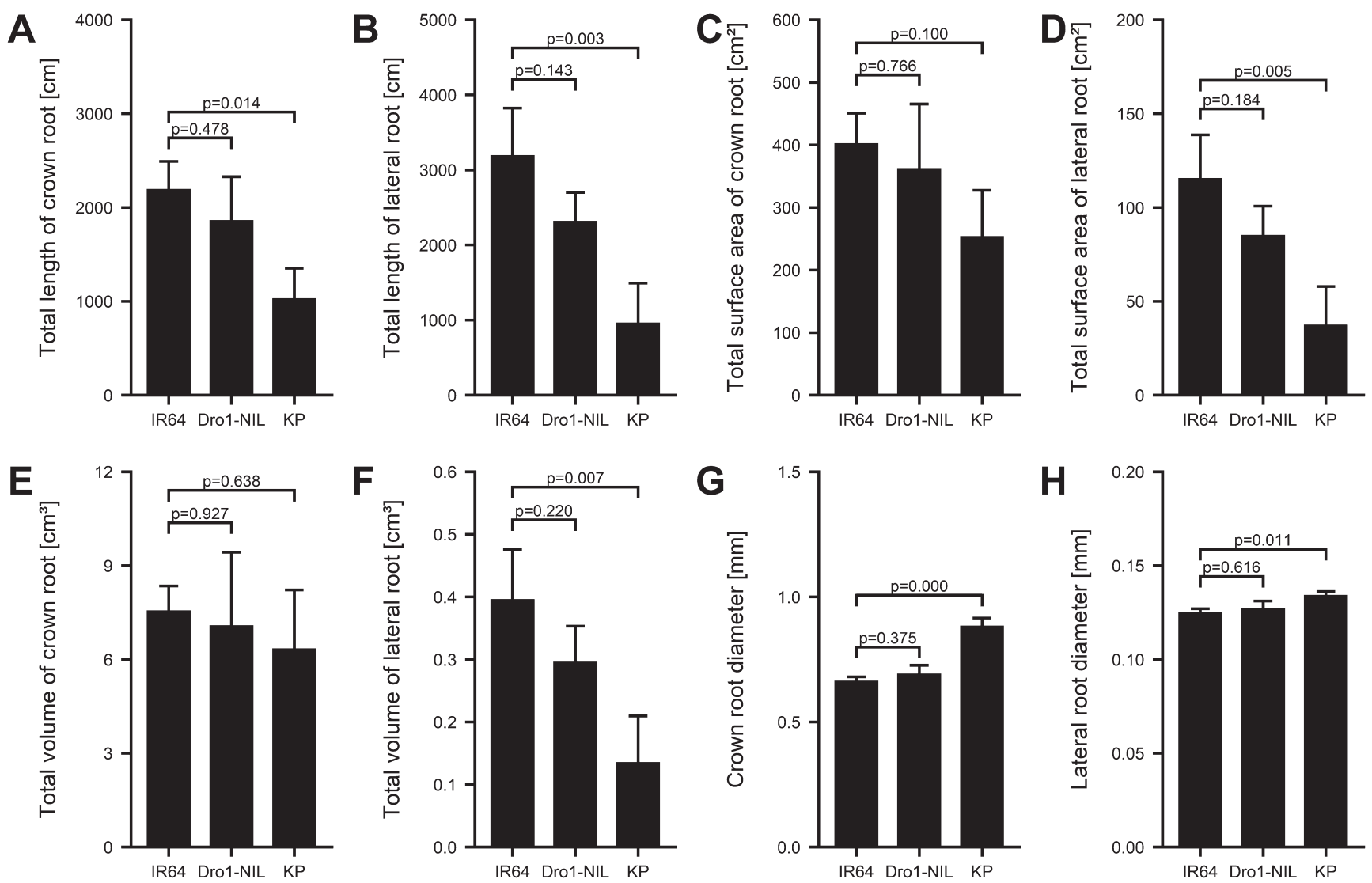

Fig. 4. Root phenotype measured with WinRHIZO ${ }^{\mathrm{TM}}$ software. Total length (A, B), total surface area (C, D), total volume (E, F), and average diameter $(\mathrm{G}, \mathrm{H})$ of crown and lateral roots were calculated from scan images of IR64, Dro1-NIL, and KP. P values calculated by Dunnett test against IR64 are shown on the figures. The number of samples of each rice line was three. Bars and error bars indicate average and standard deviation, respectively. 
(Fig. 4A, 4B). A similar pattern was observed in the total surface area and the total volume of both crown and lateral roots (Fig. 4C-4F). Compared with those of IR64 and Dro1-NIL, crown and lateral roots diameters in KP cultivar were significantly higher (Fig. 4G, 4H). We observed no significant differences of root dry weight between IR64, Dro1-NIL, and KP (Supplemental Fig. 3A), which is explained by shorter but thicker roots of KP (Fig. 4A, 4B, 4G, $4 \mathrm{H})$.

\section{Phenotype of above-ground tissues and ratio of deep root- ing}

The basket assay revealed that the RDR of Drol-NIL was significantly higher than that of IR64 (Supplemental Fig. 3B), confirming the effect of $D R O 1$ gene on deep rooting. Regarding phenotype of above-ground tissues, there were no significant differences of tiller number, plant height, and shoot dry weight between IR64 and Dro1-NIL (Supplemental Fig. 2A-2C). This result is consistent with that of a previous report demonstrating that $\mathrm{DROI}$ gene in $\mathrm{KP}$ affects only root distribution in the soil (Uga et al. 2013). On the other hand, KP showed lower tiller number, higher plant height, and higher shoot dry weight. These findings corroborate the difference between indica and tropical japonica (Gupta and O'Toole 1986).

\section{Discussion}

\section{A rapid root sampling method}

We successfully demonstrated that the backhoe-assisted monolith method is a powerful technique for collecting root samples from the plow layer in the fields. Using this method, we collected root samples of three rice lines (IR64, Dro1NIL, and KP) and found some quantitative differences on root traits. Notably, we estimated that the backhoe-assisted monolith method could collect root samples from individual plants within five minutes, which is equivalent or even quicker than the shovelomics process, which has been reported to consume about three to eight minutes per sample (Trachsel et al. 2011).

The number of persons required for the operation of this root sampling method is six: one who manipulates the backhoe, one who manipulates the loader, two who assist for handling the monolith near the backhoe and loader, and two who isolate root samples from the soil. Although at least six persons are required for efficient sampling, we conclude that the backhoe-assisted monolith method is a rapid root sampling procedure, suitable for rice root quantitative phenotyping in the fields. Furthermore, we consider that this method is also suitable for the root sampling of other crops whose cultivation involve a similar depth of the plow layer.

\section{Root sampling from the plow layer}

The depth of an upland rice plow layer is $25 \mathrm{~cm}$ at most (Gupta and O'Toole 1986) and the depth of the monolith presented in this study was $25 \mathrm{~cm}$, meaning that the backhoe- assisted monolith method is suitable for the evaluation of root properties in the plow layers. On the other hand, it is known that deep-rooting rice varieties have a high root density in soil layers deeper than $30 \mathrm{~cm}$ below the ground (Yoshida and Hasegawa 1982). Indeed, it was reported that KP elongated their roots to over $60 \mathrm{~cm}$ depth (Uga et al. 2013). Thus, the backhoe-assisted monolith method is not adequate to evaluate deep-rooting properties of rice varieties, for which other procedures such as the basket method (Oyanagi et al. 1993) or the trench method (Nemoto et al. 1998) would be suitable.

\section{Acknowledgments}

We thank the staff of the technical support center of the National Agriculture and Food Research Organization for their field management and experimental support. This work was supported by JST CREST Grant Number JPMJCR17O1, Japan.

\section{Literature Cited}

Atkinson, J.A., A. Rasmussen, R. Traini, U.Voß, C.J. Sturrock, S.J. Mooney, D.M. Wells and M.J.Bennett (2014) Branching out in roots: uncovering form, function, and regulation. Plant Physiol. 166: 538-550.

Böhm, W. (2012) Methods of studying root systems. Springer Science \& Business Media, p. 188.

Cai, H., W. Ma, X.Zhang, J.Ping, X. Yan, J.Liu, J. Yuan, L. Wang and J. Ren (2014) Effect of subsoil tillage depth on nutrient accumulation, root distribution, and grain yield in spring maize. Crop J. 2: 297-307.

Courtois, B., A.Audebert, A. Dardou, S. Roques, T. Ghneim-Herrera, G.Droc, J.Frouin, L.Rouan, E. Gozé, A.Kilian et al. (2013) Genome-wide association mapping of root traits in a japonica rice panel. PLoS ONE 8: e78037.

de Dorlodot, S., B. Forster, L. Pagès, A. Price, R. Tuberosa and X. Draye (2007) Root system architecture: opportunities and constraints for genetic improvement of crops. Trends Plant Sci. 12: 474-481.

Fageria, N.K. (2007) Yield physiology of rice. J. Plant Nutr. 30: 843879.

Food and Agriculture Organization of the United Nations (2015) World fertilizer trends and outlook to 2020, Summary report.

Gupta, P.C. and J.C. O'Toole (1986) Upland rice: a global perspective. Int. Rice Res. Inst, p. 372.

Henry,A., A.J.Cal, T.C. Batoto, R.O. Torres and R. Serraj (2012) Root attributes affecting water uptake of rice (Oryza sativa) under drought. J. Exp. Bot. 63: 4751-4763.

Hochholdinger, F., W.J. Park, M. Sauer and K. Woll (2004) From weeds to crops: genetic analysis of root development in cereals. Trends Plant Sci. 9: 42-48.

Itoh, J., K. Nonomura, K. Ikeda, S. Yamaki, Y. Inukai, H. Yamagishi, H. Kitano and Y. Nagato (2005) Rice plant development: from zygote to spikelet. Plant Cell Physiol. 46: 23-47.

Kitomi, Y., E. Nakao, S. Kawai, N. Kanno, T.Ando, S. Fukuoka, K. Irie and Y.Uga (2018) Fine mapping of QUICK ROOTING 1 and 2, quantitative trait loci increasing root length in rice. G3 (Bethesda) 8: 727-735.

Kondo, M., M.V.R. Murty and D.V.Aragones (2000) Characteristics of 
root growth and water uptake from soil in upland rice and maize under water stress. Soil Sci. Plant Nutr. 46: 721-732.

Lynch, J. (1995) Root architecture and plant productivity. Plant Physiol. 109: 7-13.

Nemoto, H., R. Suga, M. Ishihara and Y.Okutsu (1998) Deep rooted rice varieties detected through the observation of root characteristics using the trench method. Breed. Sci. 48: 321-324.

Oyanagi,A., T.Nakamoto and M.Wada (1993) Relationship between root growth angle of seedlings and vertical distribution of roots in the field in wheat cultivars. Jpn. J. Crop Sci. 62: 565-570.

Postma, J.A., A. Dathe and J.P. Lynch (2014) The optimal lateral root branching density for maize depends on nitrogen and phosphorus availability. Plant Physiol. 166: 590-602.

Slovak, R., C. Göschl, X.Su, K. Shimotani, T. Shiina and W.Busch (2014) A scalable open-source pipeline for large-scale root phenotyping of Arabidopsis. Plant Cell 26: 2390-2403.

Smith, S. and I.DeSmet (2012) Root system architecture: insights from Arabidopsis and cereal crops. Philos. Trans. R. Soc. Lond., B, Biol. Sci. 367: 1441-1452.

Song, W., B. Wang, A.L. Hauck, X. Dong, J. Li and J. Lai (2016) Genetic dissection of maize seedling root system architecture traits using an ultra-high density bin-map and a recombinant inbred line population. J. Integr. Plant Biol. 58: 266-279.

Trachsel, S., S.M.Kaeppler, K.M. Brown and J.P. Lynch (2011) Shovelomics: high throughput phenotyping of maize (Zea mays L.) root architecture in the field. Plant Soil 341: 75-87.
Uga, Y., K. Ebana, J.Abe, S. Morita, K. Okuno and M. Yano (2009) Variation in root morphology and anatomy among accessions of cultivated rice (Oryza sativa L.) with different genetic backgrounds. Breed. Sci. 59: 87-93.

Uga, Y., K. Sugimoto, S. Ogawa, J. Rane, M. Ishitani, N. Hara, Y. Kitomi, Y. Inukai, K. Ono, N.Kanno et al. (2013) Control of root system architecture by DEEPER ROOTING 1 increases rice yield under drought conditions. Nat. Genet. 45: 1097-1102.

Uga, Y., I.Assaranurak, Y.Kitomi, B.G.Larson, E.J.Craft, J.E.Shaff, J.E. Shaff, S.R. McCouch and L.V.Kochian (2018) Genomic regions responsible for seminal and crown root lengths identified by 2D \& 3D root system image analysis. BMC Genomics 19: 273.

Wasson,A.P., G.J.Rebetzke, J.A. Kirkegaard, J.Christopher, R.A. Richards and M. Watt (2014) Soil coring at multiple field environments can directly quantify variation in deep root traits to select wheat genotypes for breeding. J. Exp. Bot. 65: 6231-6249.

$\mathrm{Wu}$, J. and Y. Guo (2014) An integrated method for quantifying root architecture of field-grown maize. Ann. Bot. 114: 841-851.

Yoshida, S. and S. Hasegawa (1982) The rice root system: its development and function. In: Drought resistance in crops with emphasis on rice. Int. Rice Res. Inst. 10: 97-134.

Zhao, Y., H.Zhang, J.Xu, C. Jiang, Z. Yin, H.Xiong, J.Xie, X. Wang, X.Zhu, Y.Li et al. (2018) Loci and natural alleles underlying robust roots and adaptive domestication of upland ecotype rice in aerobic conditions. PLoS Genet. 14: e1007521. 